\title{
Rekonvaleszentenplasma zur Behandlung von schwerem COVID-19: Rationale und Design einer randomisierten, offenen klinischen Studie von Rekonvaleszentenplasma verglichen mit bestmöglicher supportiver Behandlung (CAPSID-Studie)
}

\author{
Convalescent Plasma for Treatment of Severe COVID-19: \\ Rationale and Designing of a Randomized, Open-Label Clinical \\ Trial of Convalescent Plasma Compared to Best Supportive Care \\ (CAPSID Trial)
}

\author{
Autoren \\ Institute \\ 1 Institut für Klinische Transfusionsmedizin und Immun- \\ genetik, Ulm \\ 2 Institut für Transfusionsmedizin und Immunologie, \\ Mannheim \\ 3 Institut für Transfusionsmedizin und Immunhämatologie, \\ Frankfurt am Main
}

Sixten Körper ${ }^{1}$, Bernd Jahrsdörfer ${ }^{1}$, Thomas Appl ${ }^{1}$, Harald Klüter ${ }^{2}$, Erhard Seifried ${ }^{3}$, Hubert Schrezenmeier ${ }^{1}$

Schlüsselwörter

SARS-CoV-2-Infektion, COVID-19, Rekonvaleszentenplasma, randomisierte klinische Prüfung

Key words

SARS-CoV-2 infection, COVID-19, convalescent plasma, randomized clinical trial

Bibliografie

DOI https://doi.org/10.1055/a-1090-0408

Transfusionsmedizin 2020; 10: 143-149 @ Georg Thieme

Verlag KG Stuttgart · New York | ISSN 2191-8805

Korrespondenzadresse

Prof. Dr. Hubert Schrezenmeier

Institut für Klinische Transfusionsmedizin und Immungenetik, DRK Blutspendedienst Baden-Württemberg-Hessen und Universitätsklinikum Ulm

Helmholtzstraße 10, $89081 \mathrm{Ulm}$

Tel.: + 49731150560, Fax: + 49731150500

capsid-ulm@blutspende.de

\section{ZUSAMMENFASSUNG}

Rekonvaleszentenplasma wurde bei Patienten mit akuten, viralen respiratorischen Erkrankungen mit schwerem Verlauf eingesetzt (SARS- oder MERS-Coronavirus, Influenza H1N1,
H5N1). Diese Studien erbrachten Hinweise auf eine Reduktion der Viruslast, einer Verkürzung des Krankenhausaufenthalts und eine Reduktion der Mortalität durch Rekonvaleszentenplasma. Die Wirkung konnte jedoch nicht abschließend belegt werden, da es sich nicht um randomisierte Studien handelte. Nach einer SARS-CoV-2-Infektion kommt es bei den meisten Patienten zu einer raschen Bildung von neutralisierenden Antikörpern. Wegen der pandemischen Entwicklung gibt es eine wachsende Zahl Genesener mit neutralisierenden Antikörpern, die als potenzielle Spender in Betracht kommen. Wir konzipierten eine prospektive, randomisierte Studie, um die Wirksamkeit und Sicherheit von Rekonvaleszentenplasma zur Therapie von schwerem COVID-19 und dessen Wirkmechanismus zu untersuchen (CAPSID-Studie). In diesem Beitrag werden die Rationale und das Design der Studie vorgestellt.

\section{ABSTRACT}

Convalescent plasma therapy was administered in patients with acute, viral respiratory disorders with severe clinical course (SARS- or MERS coronavirus, influenza H1N1, H5N1). These studies suggested that convalescent plasma is likely to improve viral clearance, reduce the length of hospital stay and reduce mortality and appeared to be safe. However, since controlled randomized trials were lacking only low-quality evidence was available. Most patients mount a strong humoral immune response early in the course of a SARS-CoV-2 infection, including neutralizing antibodies. Due to the pandemic an increasing number of convalescent patients who are potential donors for SARS-CoV-2 convalescent plasma will be available. We designed a prospective randomized clinical trial to study the safety and efficacy of convalescent plasma for treatment of severe COVID-19 and its mechanism of action (CAPSID trial). Here we present the rationale for this trial and major aspects of the study outline. 


\section{Einleitung}

Die Coronavirus-Krankheit 2019 (COVID-19) wird durch eine Infektion mit dem Coronavirus 2 (SARS-CoV-2) ausgelöst [1]. Die ersten Fälle wurden im Dezember 2019 in Wuhan berichtet. Am 11.03.2020 bewertete die Weltgesundheitsorganisation die zunehmende weltweite Ausbreitung als Pandemie. Trotz zahlreicher Maßnahmen kam es zu einer zunehmenden weltweiten Verbreitung mit über 11 Millionen bestätigter Fälle, über 525000 Todesfällen und weltweit mehr als 200000 Neuinfektionen pro Tag (Stand: 04.07.2020; https://covid19.who.int/). Die SARS-CoV-2Pandemie verursacht global große Herausforderungen für die Gesundheitssysteme sowie erhebliche soziale und wirtschaftliche Probleme.

Zahlreiche Studien untersuchten verschiedene pharmakologische Interventionen [2]. Einige Substanzen können den Verlauf von schwer kranken Patienten mit respiratorischer Insuffizienz positiv beeinflussen, z. B. der kürzlich in einigen Ländern vorläufig zugelassene RNA-Polymerase-Inhibitor Remdesivir, welcher in einer Studie die Zeit bis zur Erholung um 4 Tage verkürzte [3]. Ein klinischer Nutzen für andere Endpunkte, insbesondere eine Verringerung der Mortalität durch Remdesivir, ist jedoch bisher nicht belegt $[4,5]$. Die Recovery Collaboration Group berichtete kürzlich über eine Verringerung der Mortalität durch Dexamethason-Gabe bei COVID-19-Patienten mit zusätzlichem Sauerstoffbedarf oder Beatmung [6, 7]. Es gab Weiterentwicklungen im Beatmungsmanagement zur Vermeidung zusätzlicher beatmungsbedingter Lungenschäden und Empfehlungen zum Gerinnungsmanagement wegen der häufigen thromboembolischen Komplikationen [8]. Bislang konnte jedoch therapeutisch kein Durchbruch erzielt werden, der die hohe Rate schwerer Verläufe mit respiratorischer Insuffizienz und weiteren Komplikationen und die hohe Mortalität dieser Patientengruppe nachhaltig reduziert.

Die Entwicklung einer aktiven Immunisierung läuft auf Hochtouren [9] - noch gibt es aber keinen zugelassenen wirksamen Impfstoff.

\section{Warum Rekonvaleszentenplasma?}

Schon früh entstand daher die Überlegung eines Einsatzes von Rekonvaleszentenplasma bei COVID-19. Dies basierte auf folgenden Prämissen:

- Eine SARS-CoV-2-Infektion führt rasch zu einer Immunantwort mit neutralisierenden Antikörpern.

- Aufgrund der pandemischen Entwicklung einerseits und des milden Krankheitsverlaufs bei ca. $85 \%$ der Patienten, welche sich meist rasch erholen, andererseits, gibt es viele Genesene mit neutralisierenden Antikörpern, welche potenziell als Plasmaspender infrage kommen.

- Die Therapie mit gefrorenem Frischplasma ist für andere Indikationen etabliert und es gibt daher große Erfahrung mit einer Plasmatherapie, welche ein günstiges Sicherheitsprofil aufweist [10].

\section{Humorale Immunität nach SARS-CoV-2-Infektion}

Von den Epidemien mit den eng verwandten SARS- und MERS-Coronaviren war bekannt, dass es zu einer raschen und anhaltenden
Immunantwort kommt [11-24]. Diese Daten wurden zunächst auf SARS-CoV-2 extrapoliert, welches eine hohe Homologie zum SARS-Erreger (SARS-CoV) aufweist. Zwischenzeitlich werden zunehmend Daten zum Antikörperverlauf auch nach SARS-CoV-2Infektionen verfügbar: In einer Verlaufsuntersuchung bei den ersten deutschen Patienten war eine Serokonversion bereits 612 Tage nach Symptombeginn nachweisbar [25]. Sowohl IgMals auch IgG-Antikörper gegen SARS-CoV-2 sind in Immunofluoreszenzassays mit Zellen, welche das Spike-Protein von SARS-CoV-2 exprimieren, früh nachweisbar [25]. Nach 2 Wochen waren bei allen untersuchten Patienten neutralisierende Antikörper vorhanden [25]. Die Serokonversion fiel dabei mit einem langsamen, aber kontinuierlichen Abfall der Viruslast im Sputum zusammen. Auch in einer Studie aus China, welche Antikörper (IgM, $\lg G, \lg A)$ gegen das Nukleocapsid-Protein von SARS-CoV-2 mit einem ELISA (Enzyme-linked immunosorbent Assay) untersuchte, betrug das Intervall zwischen Symptombeginn und dem Nachweis von IgM- and IgA-Antikörpern nur 5 Tage. IgG-Antikörper waren 14 Tage nach Symptombeginn nachweisbar [26].

Die hohe Rate einer Serokonversion nach SARS-CoV-2-Infektion mit IgG-, IgA- und IgM-Antikörpern gegen verschiedene Epitope auf dem S-Protein und Nukleocapsid-Protein wurde in weiteren Untersuchungen bestätigt [27 - 29]. Die Titer, einschließlich Titer von neutralisierenden Antikörpern, sind interindividuell sehr variabel [27-29]. In der Untersuchung von Robbiani et al. waren nur in einem kleinen Teil der Plasmen sehr hohe Titer neutralisierender Antikörper nachweisbar [27]. Eigene Untersuchungen bestätigen die Variabilität der Antikörperantwort und zeigen auch, dass die Ergebnisse sehr vom eingesetzten Assay-System abhängen [29]. Seydoux et al. analysierten die Spezifität der Antikörper bei einem Patienten 21 Tage nach Symptombeginn [30]: Antikörper gegen die Rezeptor-bindende Domäne (RBD), welche die Interaktion zwischen dem S-Protein von SARS-CoV-2 und dem ACE2-Rezeptor hemmen, sind am stärksten neutralisierend. Die Mehrzahl der identifizierten Antikörper binden jedoch außerhalb der RBD und haben in vitro keine neutralisierende Wirkung [30]. Viele Fragen zur humoralen Immunantwort nach SARS-CoV-2-Infektion sind derzeit noch offen, insbesondere auch zur Epitopspezifität und Affinität der Antikörper, zur neutralisierenden Wirkung der Antikörper in vivo und zur Langzeitpersistenz der induzierten Antikörper. Die sequenzielle Untersuchung von Rekonvaleszentenplasmaspendern kann zur Beantwortung dieser wichtigen Fragen zur Langzeitimmunität beitragen.

\section{Frühere Erfahrung mit Rekonvaleszentenplasmen bei anderen akuten viralen respiratorischen Erkrankungen}

Rekonvaleszentenplasma wurde während der SARS- und MERSEpidemie und früheren Influenzapandemien eingesetzt [31-40]. In einer Metaanalyse von 23 Studien wurde eine statistisch signifikante Reduktion der Mortalität nach Rekonvaleszentenplasmatherapie berichtet (Odds Ratio 0,25; 95\%-Konfidenzintervall 0,14-0,45) [31]. Die Qualität der Studien in dieser Metaanalyse wurde als niedrig bis sehr niedrig bewertet und das Risiko einer Verzerrung wurde als mittel bis hoch eingestuft [31]. In Studien mit Rekonvaleszentenplasma zur Behandlung von SARS betrug die absolute Reduktion des Mortalitätsrisikos 7-23\% [31-38] 
- Tab. 1 Ein- und Ausschlusskriterien der CAPSID-Studie.

\section{Einschlusskriterien}

Patienten mit SARS-CoV-2 Infektion, für die Folgendes gilt:

1. Alter $\geq 18$ und $\leq 75$ Jahre

2. SARS-CoV-2-Infektion bestätigt durch PCR (BAL, Sputum, tiefer Nasen- oder Rachenabstrich)

3. schwerer Krankheitsverlauf, bei dem mindestens einer der unten genannten Punkte erfüllt ist:

- Atemfrequenz $\geq 30 /$ min unter Raumluft

- Atmungsunterstützung jeglicher Art

- Notwendigkeit zur intensivmedizinischen Behandlung

\section{Ausschlusskriterien}

1. Begleiterkrankung mit erwarteter Überlebenszeit unter 12 Monaten

2. nach klinischer Einschätzung infauste Prognose innerhalb der nächsten $48 \mathrm{~h}$

3. maschinelle Beatmung länger als $72 \mathrm{~h}$

4. fehlende Eligibilität für die extrakorporale Membranoxygenierung (ECMO) (auch im Falle von ARDS [Acute respiratory Distress Syndrome] gemäß Berlin-Klassifikation mit Horovitz-Index $<100 \mathrm{mg} \mathrm{Hg}$ )

5. chronisch obstruktive Lungenerkrankung (COPD), Grad 4

6. Lungenfibrose mit dem Bild einer interstitiellen Pneumonie im CT und schwerem Emphysem

7. chronische Herzerkrankung NYHA (New York Heart Association) $\geq 3$ und/oder vorbestehende Reduktion der linksventrikulären Ejektionsfraktion auf $\leq 30 \%$

8. kardiovaskuläres Versagen mit Bedarf an $\geq 0,5 \mu \mathrm{g} / \mathrm{kg} / \mathrm{min}$ Noradrenalin oder Bedarf an $>2$ vasoaktiver Medikamente über mehr als $8 \mathrm{~h}$

9. Leberzirrhose Child C

10. Leberversagen: Bilirubin > 5-Fache des Normalwerts, Erhöhung von ALT oder AST auf > 10-Fache des Normalwerts

11. jegliche positive Anamnese hinsichtlich Unverträglichkeit von Plasmaproteinen

12. bekannter IgA-Mangel

13. Schwangerschaft

14. Stillzeit

15. nicht suffizient behandelte Flüssigkeitsretention

16. Teilnahme an anderen Studien mit einer Prüfsubstanz

und die Patienten wurden früher aus der stationären Behandlung entlassen [32-34]. In einer Studie zu Rekonvaleszentenplasmabehandlung von SARS war bei den Patienten, welche Rekonvaleszentenplasma erhielten, die Wahrscheinlichkeit einer Krankenhausentlassung bis zu Tag 22 nach Therapiebeginn um 54\% höher [33].

Auch wenn diese Studien bei dem verwandten Coronavirus SARS-CoV für eine Wirkung durch Reduktion der Viruslast, Verkürzung des Krankenhausaufenthalts und Reduktion der Mortalität sprachen, konnte die Wirkung abschließend nicht belegt werden, da es sich nicht um randomisierte Studien handelte. Stattdessen schien die Anwendung von Rekonvaleszentenplasma vielfach von individueller ärztlicher Entscheidung und der Verfügbarkeit von Rekonvaleszentenplasma abzuhängen. Auch der Zeitpunkt und die Dosis der Plasmagabe sowie die Antikörper in den Rekonvaleszentenplasmen waren sehr heterogen oder wurden gar nicht erwähnt [31, 33-35, 38].

Der Einsatz von Rekonvaleszentenplasma bei Influenza (H1N1, H5N1) zeigte in Fallberichten und Fallserien positive Ergebnisse aber auch für diese Indikation gab es bisher keine randomisierten Studien [31]. Eine Metaanalyse von 6 Studien mit Rekonvaleszentenplasma, welche während des Ebolavirusausbruchs in Westafrika 2013-2016 durchgeführt wurden, kam zum Ergebnis, dass ein Vergleich der experimentellen Therapie mit nicht randomisierten Kontrollgruppen zu falschen Schlussfolgerungen führen kann [41].

\section{Warum eine randomisierte klinische Prüfung?}

Es gab im Februar 2020, dem Zeitpunkt der Konzeption der nachfolgend vorgestellten CAPSID-Studie, die Hinweise, dass der Einsatz von Rekonvaleszentenplasma bei anderen akuten viralen respiratorischen Erkrankungen wirksam und sicher sein könnte. Die von SARS und MERS bekannten Daten zeigten eine frühe, starke und anhaltende humorale Immunantwort und erste verfügbare Daten für SARS-CoV-2 bestätigten zumindest eine frühe Serokonversion. Über die Langzeitpersistenz der Antikörper konnten zu diesem Zeitpunkt noch keine Aussagen gemacht werden. Eine prospektive, randomisierte klinische Prüfung von Rekonvaleszentenplasma war jedoch trotz des wiederholten Einsatzes bei verschiedenen Epidemien/Pandemien bisher nicht durchgeführt worden. Auch wenn das Prinzip einer passiven Immunisierung überzeugend erscheint, ersetzen theoretische Überlegungen zum Wirkmechanismus nicht den Nachweis in einer randomisierten klinischen Prüfung. Offene Fragen sind unter anderem die Wirksamkeit der Antikörper in vivo, pharmakokinetische Aspekte der erreichbaren Antikörperkonzentration und -kinetik sowie die mögliche Bedeutung der Antikörpervariabilität bei rekonvaleszenten Spendern für die klinische Wirksamkeit. Auch mögliche unerwünschte Wirkungen von Rekonvaleszentenplasma müssen berücksichtigt werden: insbesondere auch TACO (Transfusion-associated circulatory Overload) und TRALI (Transfusion-related acute Lung Injury) als bekannte unerwünschte Wirkungen von Plasma. Darüber hinaus müssen auch mögliche unerwünschte Wirkungen im spezifischen Kontext einer viralen Infektion bedacht werden. Hierzu gehört das Risiko einer antikörpervermittelten Virusaufnahme in die Zelle und eine dadurch vermittelte Verschlechte- 

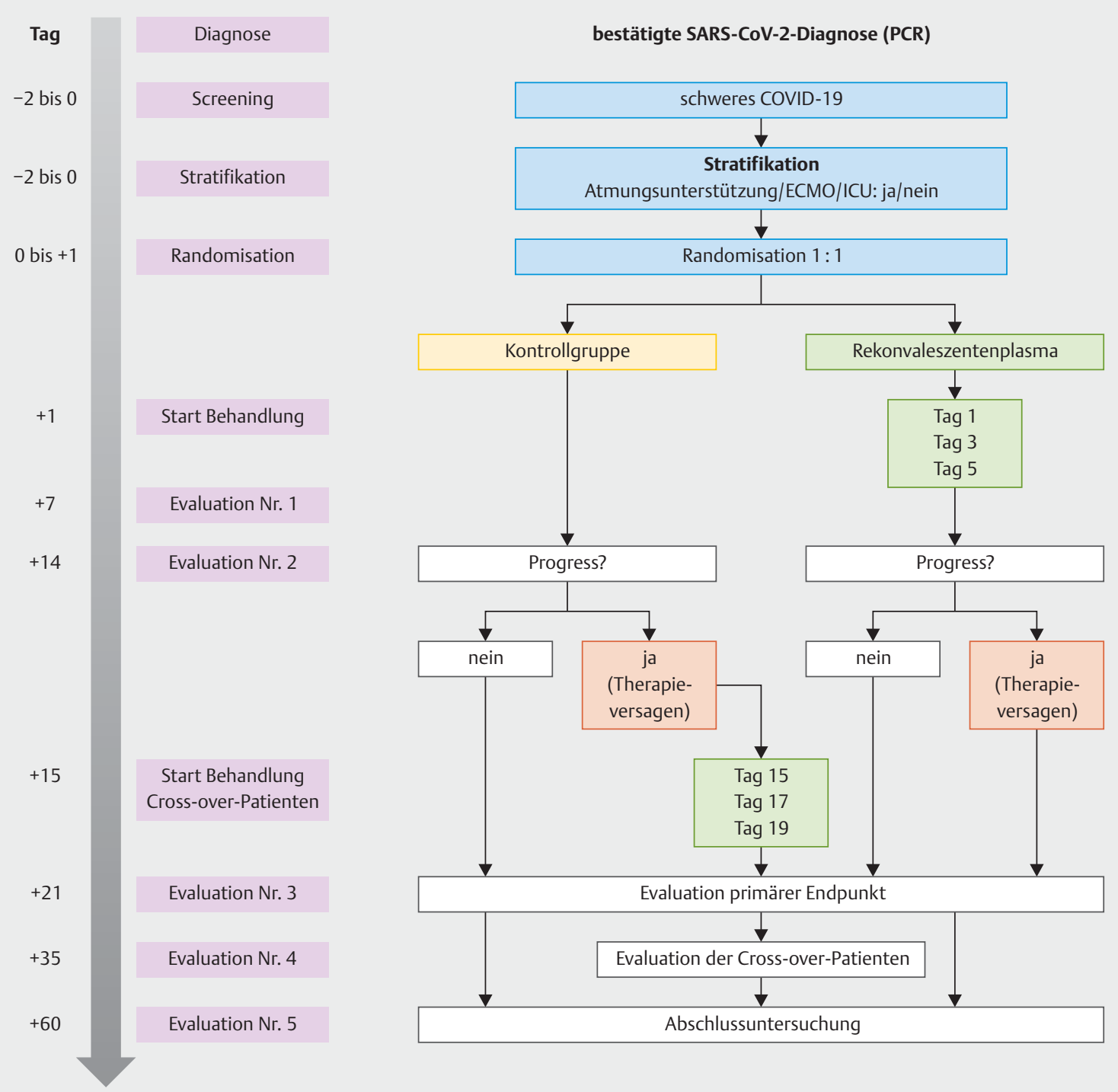

- Abb. 1 Design der CAPSID-Studie (EudraCT2020-001210-38; NCT04433910I).

rung („Antibody-dependent Enhancement“, ADE). Insbesondere bei Dengue-Virusinfektionen ist ein ADE-Phänomen wesentlich für den Krankheitsverlauf bei Reinfektionen verantwortlich [42]. Ob eine In-vitro Beobachtung, dass auch bei Coronaviren eine antikörperabhängige Virusaufnahme auftreten kann [43], auch klinisch relevant ist, kann derzeit noch nicht beurteilt werden [44]. In einem Tiermodell einer SARS-CoV-Infektion hatte die Infusion von gereinigtem Anti-Spike-Protein IgG zu einer Verschlechterung der Lungenpathologie durch Beeinflussung der Makrophagenfunktion hin zu proinflammatorischer Aktivität, unter anderem mit vermehrter Interleukin-8-Produktion, geführt [45]. Schließlich ist zu berücksichtigen, dass schweres COVID-19 durch eine Hyperinflammation und eine erhöhte Rate thromboembolischer Komplikationen charakterisiert ist. Es bedarf sorgfältiger Beobachtung, welche Auswirkungen die Gabe von Komplementund Gerinnungsfaktoren im Plasma in dieser pathophysiologischen Konstellation hat [44]. Aus den bislang vorliegenden Stu- dien bei SARS-Cov-2 wurden jedoch solche unerwünschten Wirkungen bei der Gabe von Rekonvaleszentenplasma nicht beobachtet [44-48].

Somit gibt es einerseits Hinweise auf die Wirksamkeit von Rekonvaleszentenplasma bei anderen akuten viralen respiratorischen Erkrankungen und es fehlt bisher eine andere etablierte zielgerichtete Therapie von COVID-19. Andererseits ist die Wirksamkeit und Sicherheit von Rekonvaleszentenplasma bisher nicht in einer klinischen Prüfung bestätigt worden. Sicherheitsbedenken aufgrund theoretischer Überlegungen und tierexperimenteller Befunde bedürfen einer sorgfältigen klinischen Prüfung.

Daher konzipierten wir eine prospektive, randomisierte Studie von Rekonvaleszentenplasma zur Behandlung von COVID-19. Basierend auf den Erfahrungen mit Rekonvaleszentenplasma bei SARS, MERS, Ebola, H1N1 und H5N1 sollen insbesondere auch die behandelte Patientenpopulation, der Zeitpunkt der Gabe, die Plasmadosis, der Antikörpergehalt in den Plasmen sowie die End- 
- Tab. 2 Primäre und sekundäre Endpunkte der CAPSID-Studie.

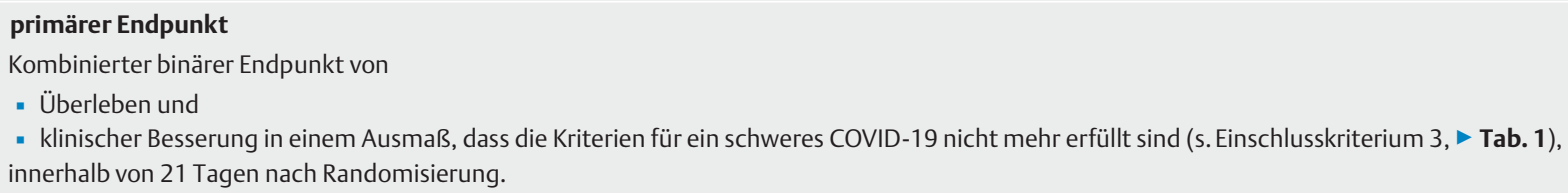

\section{sekundäre Endpunkte}

1. Zeitpunkt bis zum Erreichen der klinischen Erholung (definiert als Zeitspanne zwischen Randomisierung bis zur Verbesserung in 2 Punkten auf „WHO R\&D Blueprint seven-category ordinal Scale for clinical Improvement“) (Schlüsselsekundärendpunkt)

2. unerwünschte Ereignisse (Schlüsselsekundärendpunkt)

3. Mortalitätsrate in der Gruppe A an den Tagen 21, 35 und 60

4. Dauer der Hospitalisierung (soweit erfolgt)

5. Dauer der Intensivbehandlung (soweit erfolgt)

6. Dauer der maschinellen Beatmung/ECMO (soweit erfolgt)

7. Dauer bis negative SARS-CoV-2-PCR (tiefer Nasen- oder Rachenabstrich)

8. prädiktiver Wert der Parameter Komorbidität, Entzündung und Gerinnungsfaktoren hinsichtlich klinischer Besserung, Mortalität, Dauer der Intensivbehandlung und Dauer der Hospitalisierung

9. Machbarkeit der Plasmagewinnung von rekonvaleszenten Spendern

10. Kinetik der Anti-SARS-CoV-2-Antikörper in Plasma der Patienten = Spender nach durchgemachter SARS-Cov-2-Infektion

11. Titer der Anti-SARS-CoV-2-Antikörper in transfundierten Plasmen

12. Bedeutung folgender Spendermerkmale auf die humorale Immunität gegen SARS-CoV-2: Alter, Geschlecht, Schwere der COVID-19-Infektion, Intervall zwischen klinischer Genesung und Plasmapherese

13. Verlauf des Anti-SARS-CoV-2-Titers in beiden Gruppen zu verschiedenen Zeitpunkten nach Transfusion von Rekonvaleszentenplasmen

punkte prospektiv eindeutig definiert sein, um Aussagen zu Wirksamkeit und Sicherheit der Therapie treffen zu können.

\section{Design der CAPSID-Studie}

Die CAPSID-Studie (EudraCT2020-001210-38; NCT04433910I) ist eine prospektive, multizentrische, randomisierte Studie zur Untersuchung der Sicherheit, Wirksamkeit sowie des Wirkmechanismus von Rekonvaleszentenplasma bei COVID-19. Die Studie schließt Patienten mit schwerem COVID-19 ein. Die wesentlichen Ein- und Ausschlusskriterien sind in > Tab. 1 zusammengefasst. Diese Kriterien erfassen die Subgruppe der Patienten mit schwerem, protrahiertem Verlauf und der Gefahr von Komplikationen und hoher Mortalität.

Das Studiendesign ist in $\mathbf{A}$ Abb. 1 dargestellt: Nach Studieneinschluss erfolgt eine Stratifizierung (Atmungsunterstützung und/ oder extrakorporale Membranoxygenierung (ECMO) und/oder intensivmedizinische Behandlung), um eine Gleichverteilung der prognostisch besonders ungünstigen Subgruppe in die beiden Therapiearme zu erreichen. Anschließend erfolgt mittels der permutierten Blockrandomisationsmethode eine 1:1-Randomisation in 2 Therapiearme (Kontrollgruppe und Rekonvaleszentenplasmagruppe). Rekrutierung, Randomisation und Therapiestart sollen wegen der möglichen Dynamik der Erkrankung rasch erfolgen.

In beiden Behandlungsgruppen wird die beste derzeit etablierte Therapie verabreicht. In der Rekonvaleszentenplasmagruppe wird zusätzlich an Tag 1, 3 und 5 Rekonvaleszentenplasma (250-325 ml) appliziert. Patientinnen und Patienten in der Kontrollgruppe können bei Fortschreiten der Erkrankung in den Behandlungsarm mit Rekonvaleszentenplasma wechseln („Cross-over“). Primärer Endpunkt ist ein binärer Compound-Endpunkt aus Überleben und klinischer Besserung an Tag 21. - Tab. 2 fasst den primären Endpunkt und die wesentlichen sekundären Endpunkte zusammen.
Es ist geplant, 106 Patienten in der Studie zu behandeln. Dies basiert auf der Annahme, dass bei der eligiblen Patientengruppe der primäre Endpunkt in der Behandlungsgruppe bei $70 \%$ und in der Kontrollgruppe bei $40 \%$ der Patienten erreicht wird, bei einem $\alpha$-Fehler von 0,05 und einer Power von 0,8 .

Das Rekonvaleszentenplasma wird von Spenderinnen und Spendern gewonnen, welche von einer SARS-CoV-2-Infektion genesen sind und folgende Kriterien erfüllen:

- bestätigte Infektion mit SARS-CoV-2 (d.h. positive SARSCoV-2-PCR aus einem nasopharyngealen Abstrich in der Erkrankungsphase)

- Erholung von der SARS-CoV-2-Infektion

- Mindestintervall von 14 Tagen seit Symptomfreiheit

- kein Nachweis von SARS-CoV-2 mittels PCR in einem nasopharyngealen Abstrich und im Blut

- keine antileukozytären Antikörper (HLA-/HNA-Antikörper) (Untersuchung auf HLA-Klasse I und Klasse II und HNA-Antikörper unabhängig von Geschlecht und Schwangerschaftsanamnese)

- Nachweis neutralisierender Antikörper gegen SARS-CoV-2

Das Rekonvaleszentenplasma wird durch Plasmapherese gewonnen. Das Entnahmevolumen und die Häufigkeit der Plasmapheresen entsprechen den Vorgaben der Richtlinie Hämotherapie [46].

Das Plasma soll möglichst blutgruppengleich verabreicht werden. Um Eigenschaften der Spender bzw. der Plasmen mit dem klinischen Effekt korrelieren zu können, wird angestrebt, dass die 3 Plasmadosen eines Patienten möglichst von einem Spender kommen.

Der DRK-Blutspendedienst Baden-Württemberg - Hessen hat eine Internetseite zur Information und Registrierung spendewilliger, von SARS-CoV-2 genesener Personen eingerichtet (www. blutspende.de/rkp). 


\section{Stand und Ausblick}

Die CAPSID-Studie ist von der federführenden Ethikkommission und vielen weiteren beteiligten Ethikkommissionen in Deutschland zustimmend bewertet worden. Die Genehmigung der klinischen Prüfung gemäß § 42 AMG liegt vor. Die Studie wird vom Bundesministerium für Gesundheit unterstützt.

Die auf Hochtouren laufende Rekrutierung von Spendern für Rekonvaleszentenplasma und die Charakterisierung der SARSCoV-2-Antikörper in den Plasmen und der Verlauf der Antikörpertiter erbrachte bereits wichtige Erkenntnisse [29].

Seit Konzeption der CAPSID-Studie sind Daten von nicht randomisierten Pilotstudien $[47,48]^{*}$, einer Studie mit Fokus auf die Viruselimination nach Rekonvaleszentenplasma [49], einer großen Auswertung der Sicherheit von Compassionate-Use-Anwendungen [50] und Daten einer randomisierten Studie [48] bzw. Fallkontrollstudie [51] veröffentlicht worden. Diese Daten unterstützen das Konzept einer Rekonvaleszentenplasmatherapie, geben bisher keinen Anhalt für Sicherheitsbedenken, lassen aber andererseits noch viele Fragen offen, welche weitere Ergebnisse aus randomisierten klinischen Prüfungen erforderlich machen.

Die aus dem Zusammenwirken vieler Einrichtungen resultierende, jetzt schon sichtbare Lernkurve zu Rekonvaleszentenplasma wird für die weitere Bewertung dieser transfusionsmedizinischen Therapie sehr wichtig sein - nicht allein für SARS-CoV-2Infektionen, sondern auch als Modell für Rekonvaleszentenplasmatherapie bei anderen viralen „Newly Emerging Pathogens“.

\section{Anmerkung}

Die Rekrutierung und Studiendauer der CAPSID-Studie wird von dem weiteren Verlauf der Fallzahlen von schwerem COVID-19 in Deutschland abhängen. Die Studie wird als multizentrische Studie mit vielen beteiligten Zentren, verteilt über ganz Deutschland, durchgeführt. Weitere interessierte Zentren können mit der Studienleitung Kontakt aufnehmen, insbesondere bei SARS-CoV-2Ausbrüchen im Versorgungsgebiet (capsid-ulm@blutspende.de).

\section{Förderung}

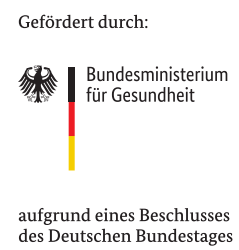

Interessenkonflikt

Die Autorinnen/Autoren geben an, dass kein Interessenkonflikt besteht.

* Siehe auch „kurz referiert“ und Kommentar in diesem Heft.
Literatur

[1] Coronaviridae Study Group of the International Committee on Taxonomy of Viruses. The species Severe acute respiratory syndrome-related coronavirus: classifying 2019-nCoV and naming it SARS-CoV-2. Nat Microbiol 2020; 5: 536-544

[2] Davis JS, Ferreira D, Denholm JT et al. Clinical trials for the prevention and treatment of COVID-19: current state of play. Med J Aust 2020. doi:10.5694/mja2.50673

[3] Beigel JH, Tomashek KM, Dodd LE et al. Remdesivir for the Treatment of Covid-19 - Preliminary Report. N Engl J Med 2020. doi:10.1056/ NEJMoa2007764

[4] Grein J, Ohmagari N, Shin D et al. Compassionate Use of Remdesivir for Patients with Severe Covid-19. N Engl J Med 2020; 382: 2327-2336

[5] Wang Y, Zhang D, Du G et al. Remdesivir in adults with severe COVID-19: a randomised, double-blind, placebo-controlled, multicentre trial. Lancet 2020; 395: 1569-1578

[6] Horby P, Lim WS, Emberson J et al. Effect of Dexamethasone in Hospitalized Patients with COVID-19: Preliminary Report. medRxiv 2020. doi:10.1101/2020.06.22.20137273

[7] Johnson RM, Vinetz JM. Dexamethasone in the management of covid19. BMJ 2020; 370: m2648. doi:10.1136/bmj.m2648

[8] Spyropoulos AC, Levy JH, Ageno W et al. Scientific and Standardization Committee Communication: Clinical Guidance on the Diagnosis, Prevention and Treatment of Venous Thromboembolism in Hospitalized Patients with COVID-19. J Thromb Haemost 2020. doi:10.1111/jth.14929

[9] O'Callaghan KP, Blatz AM, Offit PA. Developing a SARS-CoV-2 Vaccine at Warp Speed. JAMA 2020. doi:10.1001/jama.2020.12190

[10] Paul-Ehrlich-Institut; Funk MB, Heiden M, Müller S. Hämovigilanz-Bericht des Paul-Ehrlich-Instituts 2016/17: Auswertung der Meldungen von Reaktionen und Zwischenfällen nach § 63i AMG; 2019. Im Internet: https:// www.pei.de/SharedDocs/Downloads/DE/newsroom/pflichtberichte/ haemovigilanzberichte/haemovigilanz-bericht-2016-2017.pdf?_blob= publicationFile\&v=3; Stand: 23.09.2019

[11] Hsueh PR, Huang LM, Chen PJ et al. Chronological evolution of IgM, IgA, IgG and neutralisation antibodies after infection with SARS-associated coronavirus. Clin Microbiol Infect 2004; 10: 1062-1066

[12] Huang LR, Chiu CM, Yeh SH et al. Evaluation of antibody responses against SARS coronaviral nucleocapsid or spike proteins by immunoblotting or ELISA. J Med Virol 2004; 73: 338-346

[13] Woo PC, Lau SK, Wong BH et al. Longitudinal profile of immunoglobulin $G(\lg G), \lg M$, and $\lg A$ antibodies against the severe acute respiratory syndrome (SARS) coronavirus nucleocapsid protein in patients with pneumonia due to the SARS coronavirus. Clin Diagn Lab Immunol 2004; 11: 665-668

[14] Chen W, Xu Z, Mu J et al. Antibody response and viraemia during the course of severe acute respiratory syndrome (SARS)-associated coronavirus infection. J Med Microbiol 2004; 53: 435-438

[15] Chen X, Zhou B, Li M et al. Serology of severe acute respiratory syndrome: implications for surveillance and outcome. J Infect Dis 2004; 189: $1158-1163$

[16] Mo H, Zeng G, Ren X et al. Longitudinal profile of antibodies against SARS-coronavirus in SARS patients and their clinical significance. Respirology 2006; 11: 49-53

[17] Shi Y, Wan Z, Li L et al. Antibody responses against SARS-coronavirus and its nucleocaspid in SARS patients. J Clin Virol 2004; 31: 66-68

[18] Qiu M, Shi Y, Guo Z et al. Antibody responses to individual proteins of SARS coronavirus and their neutralization activities. Microbes Infect 2005; 7: 882-889

[19] Lee N, Chan PK, Ip M et al. Anti-SARS-CoV IgG response in relation to disease severity of severe acute respiratory syndrome. J Clin Virol 2006; 35 : 179-184 
[20] Zhao J, Wang W, Wang W et al. Comparison of immunoglobulin G responses to the spike and nucleocapsid proteins of severe acute respiratory syndrome (SARS) coronavirus in patients with SARS. Clin Vaccine Immunol 2007; 14: 839-846

[21] Yang Z, Wang S, Li Q et al. Determining SARS sub-clinical infection: a longitudinal seroepidemiological study in recovered SARS patients and controls after an outbreak in a general hospital. Scand J Infect Dis 2009; 41: 507-510

[22] Li G, Fan Y, Lai Y et al. Coronavirus infections and immune responses. J Med Virol 2020; 92: 424-432

[23] Park WB, Perera RA, Choe PG et al. Kinetics of Serologic Responses to MERS Coronavirus Infection in Humans, South Korea. Emerg Infect Dis 2015; 21: 2186-2189

[24] Choe PG, Perera RAPM, Park WB et al. MERS-CoV Antibody Responses 1 Year after Symptom Onset, South Korea, 2015. Emerg Infect Dis 2017; 23: 1079-1084

[25] Wölfel R, Corman VM, Guggemos W et al. Virological assessment of hospitalized cases of coronavirus disease 2019. Nature 2020; 581: 465-469

[26] Guo L, Ren L, Yang S et al. Profiling Early Humoral Response to Diagnose Novel Coronavirus Disease (COVID-19). Clin Infect Dis 2020. doi:10.1093/cid/ciaa310

[27] Robbiani DF, Gaebler C, Muecksch F et al. Convergent antibody responses to SARS-CoV-2 in convalescent individuals. Nature 2020. doi:10.1038/s41586-020-2456-9

[28] Liu W, Liu L, Kou G et al. Evaluation of Nucleocapsid and Spike ProteinBased Enzyme-Linked Immunosorbent Assays for Detecting Antibodies against SARS-CoV-2. J Clin Microbiol 2020. doi:10.1128/JCM.00461-20

[29] Jahrsdorfer B, Kroschel J, Ludwig C et al. Independent side-by-side validation and comparison of four serological platforms for SARS-CoV-2 antibody testing. 2020 [submitted]

[30] Seydoux E, Homad L], MacCamy A] et al. Analysis of a SARS-CoV-2-Infected Individual Reveals Development of Potent Neutralizing Antibodies with Limited Somatic Mutation. Immunity 2020. doi:10.1016/j. immuni.2020.06.001

[31] Mair-Jenkins ], Saavedra-Campos M, Baillie JK et al. The effectiveness of convalescent plasma and hyperimmune immunoglobulin for the treatment of severe acute respiratory infections of viral etiology: a systematic review and exploratory meta-analysis. J Infect Dis 2015; 211: 80-90

[32] Zhou XZ, Zhao M, Wang FS et al. [Epidemiologic features, clinical diagnosis and therapy of first cluster of patients with severe acute respiratory syndrome in Beijing area]. Zhonghua Yi Xue Za Zhi 2003; 83: 1018-1022

[33] Soo YO, Cheng Y, Wong R et al. Retrospective comparison of convalescent plasma with continuing high-dose methylprednisolone treatment in SARS patients. Clin Microbiol Infect 2004; 10: 676-678

[34] Cheng Y, Wong R, Soo YO et al. Use of convalescent plasma therapy in SARS patients in Hong Kong. Eur J Clin Microbiol Infect Dis 2005; 24: 44-46

[35] Wong SS, Yuen KY. The management of coronavirus infections with particular reference to SARS. J Antimicrob Chemother 2008; 62: 437-441
[36] Nie QH, Luo XD, Hui WL. Advances in clinical diagnosis and treatment of severe acute respiratory syndrome. World J Gastroenterol 2003; 9: $1139-1143$

[37] Wong VW, Dai D, Wu AK et al. Treatment of severe acute respiratory syndrome with convalescent plasma. Hong Kong Med J 2003; 9: 199-201

[38] Yeh KM, Chiueh TS, Siu LK et al. Experience of using convalescent plasma for severe acute respiratory syndrome among healthcare workers in a Taiwan hospital. J Antimicrob Chemother 2005; 56: 919-922

[39] Lai ST. Treatment of severe acute respiratory syndrome. Eur J Clin Microbiol Infect Dis 2005; 24: 583-591

[40] Arabi YM, Hajeer AH, Luke T et al. Feasibility of Using Convalescent Plasma Immunotherapy for MERS-CoV Infection, Saudi Arabia. Emerg Infect Dis 2016; 22: 1554-1561

[41] Dodd LE, Follmann D, Proschan M et al. A meta-analysis of clinical studies conducted during the West Africa Ebola virus disease outbreak confirms the need for randomized control groups. Sci Transl Med 2019; 11: 11520

[42] Katzelnick LC, Gresh L, Halloran ME et al. Antibody-dependent enhancement of severe dengue disease in humans. Science 2017; 358: 929-932

[43] Wan Y, Shang J, Sun S et al. Molecular Mechanism for Antibody-Dependent Enhancement of Coronavirus Entry. J Virol 2020. doi:10.1128/ JVI.02015-19

[44] Dzik S. COVID-19 Convalescent Plasma: Now Is the Time for Better Science. Transfus Med Rev 2020. doi:10.1016/j.tmrv.2020.04.002

[45] Liu L, Wei Q, Lin Q et al. Anti-spike IgG causes severe acute lung injury by skewing macrophage responses during acute SARS-CoV infection. JCI Insight 2019. doi:10.1172/jci.insight. 123158

[46] Vorstand der Bundesärztekammer auf Empfehlung seines Wissenschaftlichen Beirats und im Einvernehmen mit dem Paul-Ehrlich-Institut. Richtlinie zur Gewinnung von Blut und Blutbestandteilen und zur Anwendung von Blutprodukten (Richtlinie Hämotherapie). Köln: Deutscher Ärzteverlag; 2017

[47] Duan K, Liu B, Li C et al. Effectiveness of convalescent plasma therapy in severe COVID-19 patients. Proc Natl Acad Sci U S A 2020; 117: 94909496

[48] Shen C, Wang Z, Zhao F et al. Treatment of 5 Critically III Patients with COVID-19 with Convalescent Plasma. JAMA 2020; 323: 1582-1589

[49] Zeng QL, Yu Z], Gou J] et al. Effect of Convalescent Plasma Therapy on Viral Shedding and Survival in Patients with Coronavirus Disease 2019. J Infect Dis 2020; 222: 38-43

[50] Joyner M], Wright RS, Fairweather D et al. Early safety indicators of COVID-19 convalescent plasma in 5,000 patients. J Clin Invest 2020. doi:10.1172/JCl140200

[51] Liu STH, Lin HM, Baine I et al. Convalescent plasma treatment of severe COVID-19: A matched control study. medRxiv 2020. doi:10.1101/ 2020.05.20.20102236 